\title{
DISCUSSION
}

\section{The effects of pressuremeter geometry on the results of tests in clay}

\author{
G. T. HOULSBY and J. P. CARTER (1993). Géotechnique 43, No. 4, 567-576
}

\section{G. Jefferies, Golder Associates (UK) Ltd}

The geometry of the self-bored pressuremeter (SBP), as characterized by its length to diameter ratio $L / D$, can undoubtedly bias the soil properties estimated from SBP data. The question is: by how much? In terms of the undrained strength the authors state that 'the strength derived from the pressuremeter would be about $25-43 \%$ higher than the actual strength of the soil'. I would argue that the magnitude of strength bias suggested by the authors is about three times larger than the actual bias; the actual bias due of $L / D$ effects is of the order of $10 \%$ for commonly available SBPs.

$\Lambda$ limit for $L / D$ effects can be computed from the expansion of a cavity in an infinite elasticplastic (Tresca) material-the same material as considered in the paper. The cylindrical cavity has the least kinematic freedom of a cavity expanded in three-dimensional space; a spherical cavity has the most kinematic freedom. Thus these two conditions should bound the kinematic freedom experienced by a finite length pressuremeter. The now well-known solution for the cavity pressure at cavity radius $a$ expanded from an initial radius $a_{0}$ is

$$
\frac{\psi-\sigma_{\mathrm{ho}}}{s_{\mathrm{u}}}=\frac{2+m}{3}\left\{1+\ln \left[I_{\mathrm{r}}\left(1-A^{m+1}\right)\right]\right\}
$$

where $A=a_{0} / \alpha$ and the integer $m=1,2$ is used as a toggle to switch between cylindrical and spherical solutions respectively (following the notation of Houlsby \& Withers, 1988). For a given value of $\psi-\sigma_{\mathrm{h} 0}$ and $A$, the smallest strength will be computed from the spherical solution (equation (4) with $m=2$ ) which conceptually corresponds to $L / D \sim 1$. If this spherically derived strength is denoted by $s_{3}$, then $s_{3} \leqslant s_{\mathrm{u}}$ because spherical conditions provide the least kinematic constraint for the cavity expansion. Further, suppose that one is ignorant of the geometric limitations of the SBP and the data are interpretcd using the cylindrical theory (equation (4) with $m=1$ ), as is conventionally done, with the strength obtained being $s_{u m}$. Then it follows that maximum correction factor is given by the ratio $s_{3} / s_{\text {um }}$, which is readily obtained from equa- tion (4). This limiting ratio $s_{3} / s_{\mathrm{um}}$ is plotted as a function of $I_{\mathrm{r}}$ in Fig. 11 against the computed trends reported by the authors, using $A=0.96$ which lies within their strain interval for strength determination. Although the limiting correction ratio varies slightly with $A$, this variation does not affect the substantive result: the $L / D$ correction factors proposed in the paper exceed the limiting condition from theoretical solutions at large rigidity. It seems that there is some error with the reported numerics.

Apart from difficulties with the numerics, the real correction factor will still be less than is suggested by the authors because of a misunderstanding about rigidity in the Tresca model. The elastic-perfectly plastic (Tresca) model represents the stress-strain behaviour of clay as bilinear, the sloping portion of which can include workhardening plastic strain. It does not follow that a truly elastic $G$ should be used to estimate rigidity. This is most easily seen by example.

All in situ tests present inverse boundary value problems and in the case of the SBP unique solutions can be obtained only by using expansion and contraction data. Essentially identical theoretical approaches to do this are given by Iloulsby \& Withers (1988) and Jefferies (1988), these approaches being adequate to constrain $s_{u}$ typically within a few per cent (which is as much as one can expect with an inverse problem). Fig. 12(a) shows the fit of the complete solution to an SBP test in London clay, from which it can be seen that $G$ used to model the test is about $25 \%$ of the elastic value found in unload-reload cycles. If an elastic $G$ is used in the cavity expansioncontraction theory, then the Tresca model cannot be fitted to the data as shown on Fig. 12(b) for the same test data.

SBP tests have now been analysed using complete expansion-contraction theory in many clays. Some of this experience is summarized in Table 7 in terms of the required $I_{\mathrm{r}}$ to fit the test data with a Tresca model; both the range and the most frequent (typical) value are shown. Experience suggests that the typical range of rigidity is $70<I_{\mathrm{r}}<180$ not $250<I_{\mathrm{r}}<800$ as suggested in the paper. The importance of this understanding 


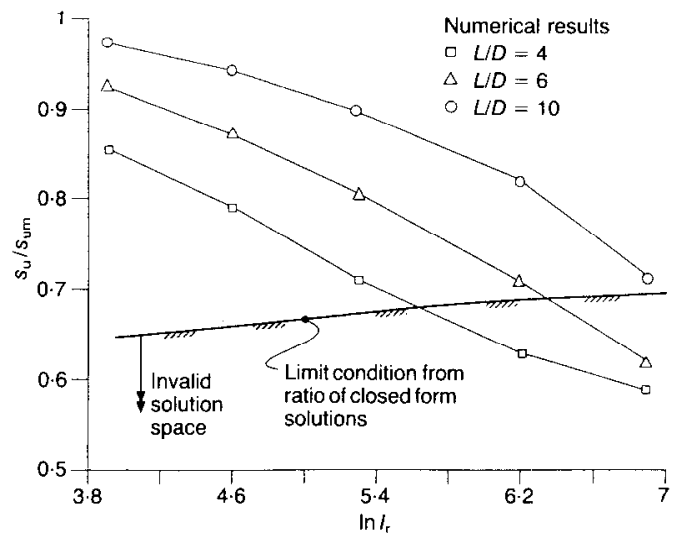

Fig. 11. Variation of strength correction factor with $L / D$ ratio and rigidity index : central strain measurement

Table 7. Examples of $I_{r}$ to model SBP data

\begin{tabular}{l|c|c}
\hline \multicolumn{1}{c|}{ Clay } & $I_{\mathrm{r}}$ range & Typical $I_{r}$ \\
\hline Boston Blue & $120-160$ & 150 \\
\hline London & $100-250$ & 180 \\
\hline Bothkennar & $90-160$ & 110 \\
\hline $\begin{array}{l}\text { Prairie Valley } \\
\text { (Alberta) }\end{array}$ & $120-190$ & 150 \\
\hline $\begin{array}{l}\text { Beaufort Shelf, } \\
\text { Kringalik Plateau }\end{array}$ & $85-180$ & 145 \\
\hline $\begin{array}{l}\text { Beaufort Shelf, } \\
\text { Amauligak Channel }\end{array}$ & $55-180$ & 70 \\
\hline
\end{tabular}

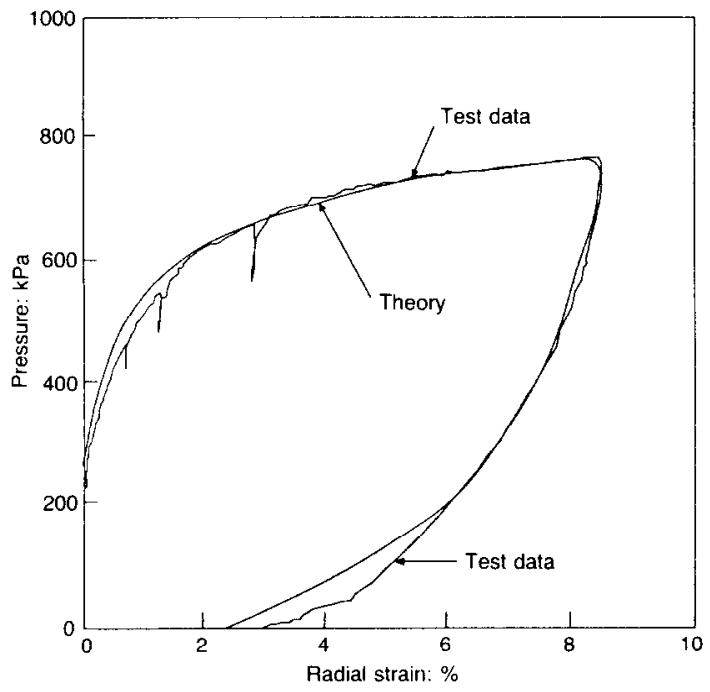

(a) of $I_{\mathrm{r}}$ in clay follows immediately from Fig. 6. Using $70<I_{\mathrm{r}}<180$ gives $0.82<s_{\mathrm{u}} / s_{\mathrm{um}}<0.90$, which can be expressed as the strength derived from the pressuremeter using cylindrical theory may be $10-18 \%$ greater than the true value. This is about one third of the bias suggested in the papcr.

The concept of bias automatically implies that there is a corresonding concept of some reference value. In the paper, this reference value is known because it is specified as the input. However, concern about SBP-derived $s_{\mathrm{u}}$ arose in field situations from comparison with other measures of undrained strength (which thus involves several more issues on bias than just $L / D$ effects), as noted by the authors. A common strength used in such comparisons is the triaxial compression strength.

There is an innocent simplicity in referring to an undrained triaxial strength as if this were a sufficient definition to assure reasonable uniqueness. Both the sampling method and test protocol can makc a substantial difference to the measured clay behaviour even when the triaxial test is restricted to conventional compression loading. The importance of sampling and protocol factors can be judged from recent research at the Bothkennar site. Fig. 13 shows $s_{u}$ profiles obtained with each of five sampling/testing protocols. The trends in Fig. 13 have been developed by fitting the best straight line through the data summary reported by Hight, Bond \& Legge (1992); thus Fig. 13 represents the true uncertainty in $s_{u}$ as soil variability effects have been averaged out. There is at least as much uncertainty in the undrained

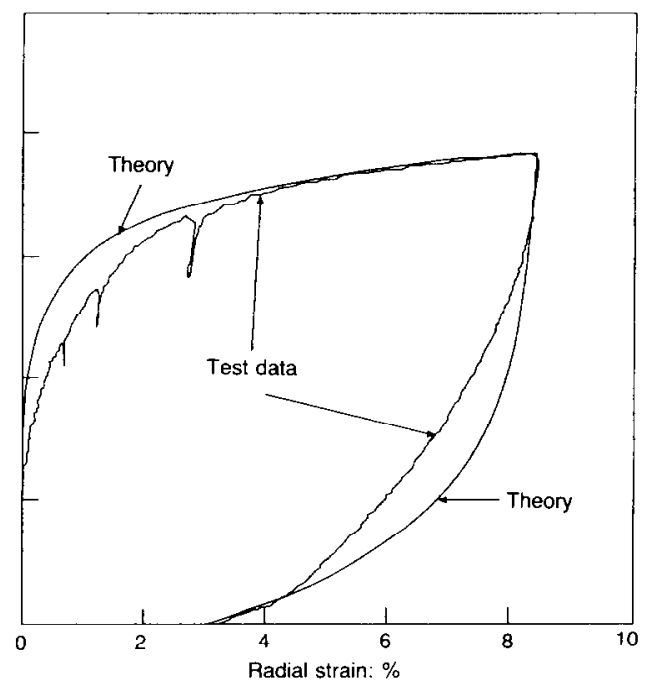

(b)

Fig. 12. Comparison of theory with measured SBP data for alternative idealizations of $I_{\mathrm{r}}$ (London clay): (a) $I_{\mathrm{r}}$ treated as pseudo-elastic and selected for best-fit of theory to data; (b) $I_{\mathrm{r}}$ estimated from elastic unloading 
triaxial compression strength concept itself as any systematic bias between the SBP and the triaxial test.

The Bothkennar research also included SBP tests, and the reported $s_{u}$ profile (smoothed to remove variability) is plotted against the uncertain triaxial data in Fig. 14. As there are several approaches to estimating $s_{u}$ from the SBP, the original site data were accessed and fitted with computer-aided modelling to check the reported strengths using full expansion-contraction analysis; similar values of $s_{u}$ to those reported by Hight et al. (1992) were found. As can be seen, the SBP strengths, uncorrected for any $L / D$ bias, lie inside the uncertainty of the triaxial data, although with a slightly different trend against depth. A similar one for one correspondence between triaxial and SBP strengths was also shown in fig. 9 of Houlsby \& Withers (1988).

Elastic modulus data are available at Bothkennar from seismic shear wave measurements (Hight et al., 1992). This modulus profile combines with the uncorrected SBP $s_{u}$ profile to give rigidities of about $I_{\mathrm{r}}=650$. Using this rigidity and the correction chart proposed in the paper

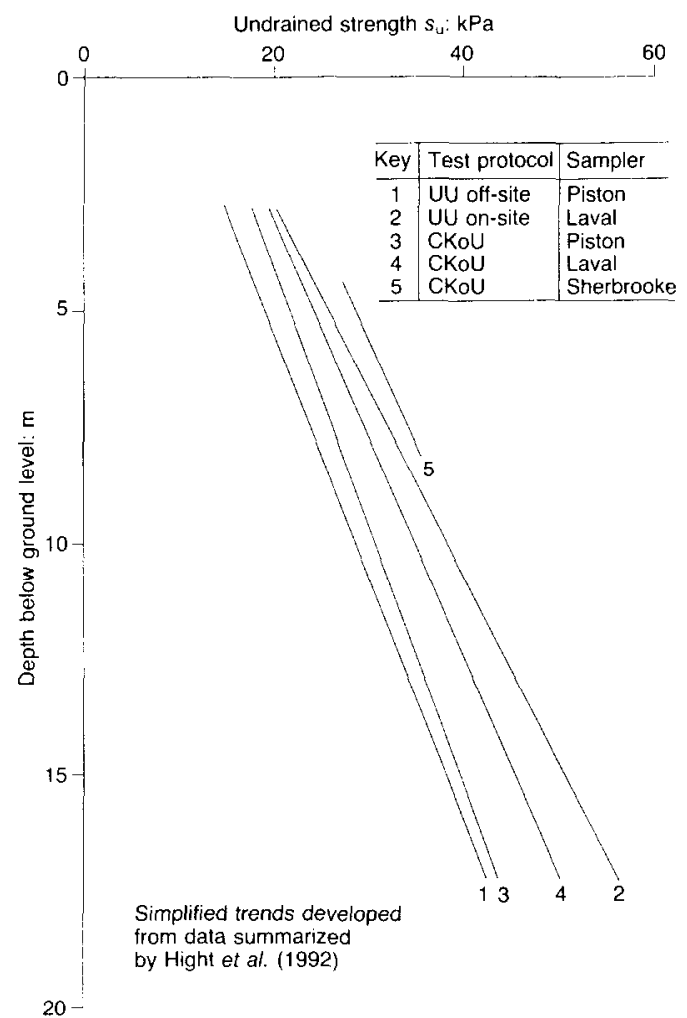

Fig. 13. Systematic bias in triaxial compression undrained strength profiles at Bothkennar gives a much reduced strength profile which is also shown in Fig. 14. The reduced strengths from the SBP are as little as half those that might be estimated from triaxial testing, further confirming that the proposed combination of elastic rigidity and $L / D$ correction is grossly conservative.

Check modelling of the SBP data at Bothkennar was carried out and gave self-consistent rigidities of typically about $I_{\mathrm{r}}=110$. Such rigidity leads to a correction factor of about $10 \%$ from Fig. 6 and this reduced strength profile is also shown in Fig. 14. It lies towards the lower bound of the uncertainty in strength from the triaxial data and essentially maps to strengths derived from piston samples. This is still a lower strength than expected as, on theoretical grounds (because of the intermediate principal stress) it would be usual to have a slightly higher strength from the SRP than the triaxial test, all else being equal.

In conclusion, there must be a systematic bias in $s_{\mathrm{u}}$ derived from the SBP using cylindrical cavity theory because of finite pressuremeter length, and the authors' suggestion of a rigiditybased bias correction of $s_{\mathrm{u}}$ is an acceptable engineering approximation. However, based on a

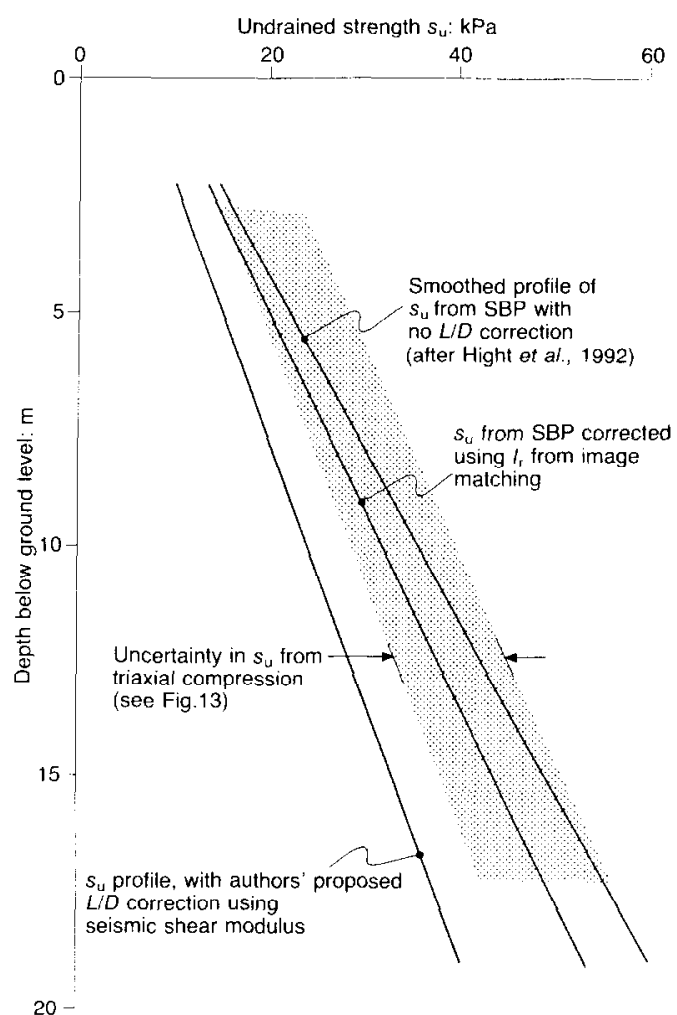

Fig. 14. Comparison of $s_{\mathrm{u}}$ from SBP with triaxial data at Bothkennar 
consideration of closed-form solutions, the bias computed by the authors' numerics seems larger than is reasonable. Further, the rigidity used in estimating bias correction must not be computed from an elastic shear modulus but must use the rigidity required to model the complete expansion-contraction of the SBP test data. The understanding of rigidity on its own suggests that the strength bias is about $10-18 \%$-less than half that suggested by the authors. Given the concern about the numerics, it is reasonable to expect that true bias is towards the lower end of the range (say about $10 \%$ ), which is about a third of that proposed by the authors. The estimation that $L / D$ bias is about $10 \%$ is reinforced by consideration of the Bothkennar research data. There can also be other effects which influence measured SBP data, such as strain rate; these effects will be common to both SBP and any other test. However, test standardization and more advanced constitutive models are required before such issues can be discussed meaningfully. Accordingly, for the present, it appears that $s_{u}$ derived from the SBP using diligent analysis of both expansion and contraction data, with a modest correction for $L / D$ bias, is at least as reliable as a strength obtained with the very best sampling and laboratory triaxial testing. The SBP is also more convenient as it uses real time results.

I am grateful to D. Nash of Bristol University for a copy of the original SBP test data from the Bothkennar site.

\section{A. Shuttle, Golder Associates Inc.}

The authors' numerical modelling undoubtedly indicates that the effect on pressuremeter pressure of the end restraint due to finite $L / D$ is not negligible and needs to be considered during interpretation. However, they state that 'the strength derived from the pressuremeter would be about

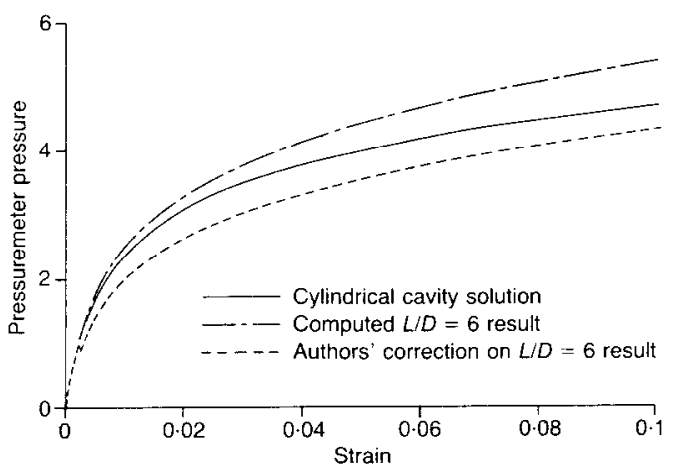

Fig. 15. Pressure plotted against strain for $I_{\mathrm{r}}=200$
$25-43 \%$ higher than the actual strength of the soil'. I would suggest that the magnitude of the errors is a direct result of the interpretation method, that the strain range used for the $\psi$ versus $\ln (\varepsilon)$ fit will affect the correction factor, and that the true overshoot is much smaller than the authors suggest, the true error in the rcsulting pressures being in the range $2-17 \%$ for a typical pressuremeter geometry of $L / D \approx 6$.

The paper suggests that a correction factor, which is constant for any soil rigidity, can be applied to $s_{\mathrm{u}}$ to account for the effect of finite geometry in the SBP pressure-expansion relationship. Defining this correction as $\beta$ gives

$$
\left[\psi-\sigma_{\mathrm{h} 0}\right]_{L / D=\infty}=\beta\left[\psi-\sigma_{\mathrm{ho}}\right]_{L / D=n}
$$

or

$$
s_{\text {u(true })}=\beta s_{\text {u(measured })}
$$

where $n$ is the geometric factor of the SBP, which is typically 6 for many commercial devices; all else remains fixed. In this discussion, correcting SBP data by $\beta$ is referred to as an overshoot correction.

Figure 4 shows that $\beta$ is not a constant but varies with cavity strain. It is thus unclear that determining a strength correction factor for recovering $s_{\mathrm{u}}$ estimated from the slope of a $\psi$ versus $\ln (\varepsilon)$ plot is consistent with the authors' hypothesis.

To look more closely at correction factors for finite length SBP tests in clay, numerical simulations were reproduced for $L / D=6$ using an adaption of a University of Manchester finite element program (Smith \& Griffiths, 1988). With this code the viscoplastic solution method resulted in errors in the computed $s_{\mathrm{u}}$ values of an infinite cylinder using the $\psi$ versus $\ln (\varepsilon)$ approach of less than $1 \%$ for the full range of $I_{\mathrm{r}}$, hence allowing direct calculation of correction factors from the numerical simulations without the diffi-

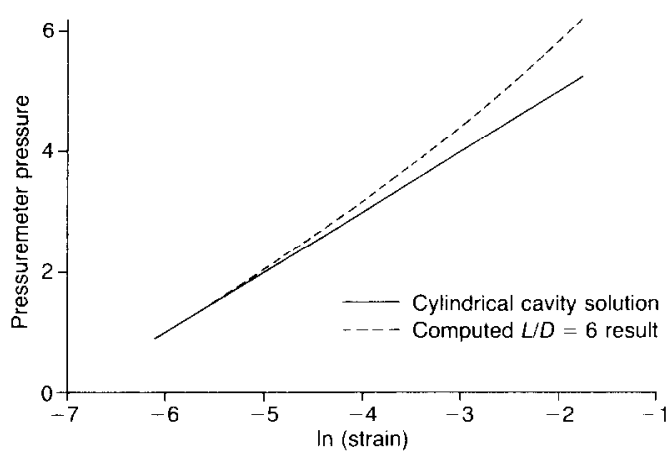

Fig. 16. Pressure plotted against $\ln \left(\right.$ strain), $I_{r}=200$ 
culties discussed by the authors. For compatibility, the same fixity above the pressuremeter was used as is understood to have been used in the paper. Both calculation and the reference cavity solution were small strain.

The results of the modelling indicated similar slopes in the $\psi$ versus $\ln (c)$ plot over the range $2-5 \%$ strain as those achieved by the authors. However, the resulting corrections were not, as the authors suggested, relatively insensitive to the choice of strain range chosen. Fig. 15 compares the infinite cylinder and finite $L / D=6$ pressuremeter response corrected over the entire yielded strain range using the $\psi$ versus $\ln (\varepsilon)$ correction for $2-5 \%$ strain with $I_{\mathrm{r}}=200$. It is shown that the correction factor derived from the $\psi$ versus $\ln (\varepsilon)$ fit is extremely poor at mapping the $L / D=6$ solution to cylindrical cavity theory. In fact, the authors proposed correction is about twice the fit for $\beta$.

The nature of the difficulty with the authors' approach can be seen in Fig. 16 which compares the infinite cylinder and finite $L / D=6$ pressuremeter in $\ln (\varepsilon)$ space, again using $I_{\mathrm{r}}=200$. This shows that the slope error increases with strain over the range of pressures considered. This is further illustrated in Table 8, which shows the calculated correction factors for a relatively small range of strain intervals between yield and 7\% strain, typically with a range of $3 \%$. The difference in the correction factors varies by $7.5 \%$ at $I_{\mathrm{r}}=50$, to $15 \%$ at $I_{\mathrm{r}}=200$ and $16 \%$ at $I_{\mathrm{r}}=1000$, this being of the same order as the correction factor itself at lower values of rigidity.

The conventional $\psi$ versus $\ln (\varepsilon)$ approach also results in higher correction factors than are consistent with correcting for $L / D$ using $s_{\mathbf{u}}$. The differences between overshoot and $\log _{e}$ derived correction factors are summarized in Fig. 17 where the correction factors derived using the $\psi$ versus $\ln (\varepsilon)$ method in the $2-5 \%$ strain range are compared with the actual overshoot correction factor for the pressuremeter pressure at both $2 \%$ and $5 \%$ strain. The actual discrepancy between the computed $L / D=6$ and theoretical solution is much smaller than the slope of the $\log _{e}$ fit. At the

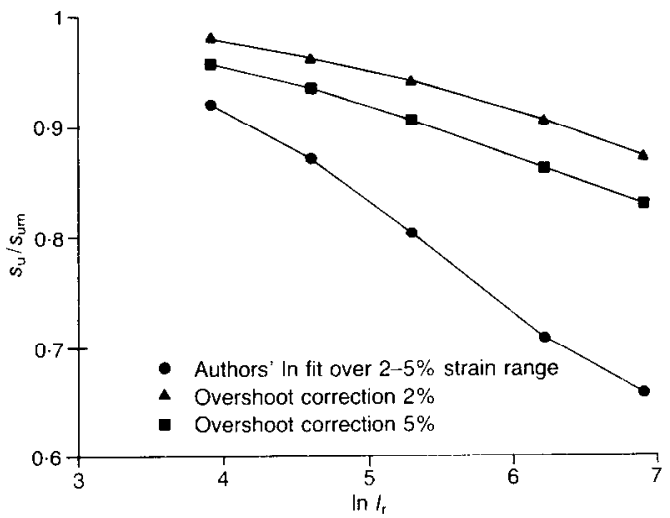

Fig. 17. Correction to $L / D=6$ solution using Overshoot and $\ln$ (strain) method

upper end of the $I_{\mathrm{r}}$ range considered, which is higher than normally encountered in practice, the error is $13 \%-17 \%$ compared with the $\log _{\mathrm{c}}$ slope correction of $34 \%$.

The effects of finite pressuremeter length require more attention to ensure that the analysis method provides the data required for the design process. However, although there is certainly a correlation between rigidity index and the correction factor for $s_{\mathrm{u}}$ for a finite length pressuremeter, the correction factors computed using the slope of the $\psi$ versus $\ln (\varepsilon)$ graph are strain-dependent and suggest strengths overestimates for the pressuremeter which are not necessarily justified if alternative analysis methods such as image matching are used. However, the length dependence of the pressuremeter response at $L / D \approx 6$ strongly suggests that the coefficient of earth pressure at rest $K_{0}$ will also affect the response. Further work on this subject is required.

\section{Reply by G. T. Houlsby}

The discussion by Jefferies contains a number of important errors and misunderstandings and obscures the discussion by adopting an unnecessarily aggressive approach in attempting to refute the conclusions in the paper.

Table 8. Correction factor for $L / D=6$ derived from fit to $\psi$ versus ln(s) curve

\begin{tabular}{c|c|c|c|c|c}
\hline \multirow{2}{*}{$\begin{array}{c}\text { Strain } \\
\text { range }\end{array}$} & \multicolumn{5}{|c}{$I_{\mathrm{r}}$} \\
\cline { 2 - 6 } & 50 & 100 & 200 & 500 & 1000 \\
\hline Yicld to 3\% & 0.9620 & 0.9335 & 0.9016 & 0.8606 & 0.8257 \\
$1-4 \%$ & 0.9444 & 0.9041 & 0.8460 & 0.7553 & 0.6879 \\
$2-5 \%$ & 0.9203 & 0.8707 & 0.8027 & 0.7067 & 0.6562 \\
$3-6 \%$ & 0.9021 & 0.8424 & 0.7755 & 0.6839 & 0.6511 \\
$4-7 \%$ & 0.8866 & 0.8260 & 0.7519 & 0.6662 & 0.6687 \\
\hline
\end{tabular}


Jefferies starts by attempting to show that there is inconsistency in the analysis in the paper. $\mathrm{He}$ quotes a correct solution for cylindrical and spherical cavity expansion, which for the small strain analysis presented in the paper reduces to

$$
\frac{\psi-\sigma_{\mathrm{ho}}}{s_{\mathrm{u}}}=\frac{2+m}{3}\left\{1+\ln \left[(m+1) I_{\mathrm{r}} \varepsilon\right]\right\}
$$

By choosing $\varepsilon=0.04$ Jefferies then shows that (if $\psi, I_{\mathrm{r}}$ and $\sigma_{\mathrm{h} 0}$ are known) a spherical expansion interpreted using a theory appropriate to the cylindrical case would result in an overestimate of the undrained strength of about 31-35\%. As he regards (unjustifiably) the spherical cavity expansion pressure as a theoretical upper limit, he interprets this result as an upper bound on the results, and labels all results indicating a greater overestimation of undrained strength as 'Invalid solution space' in Fig. 11.

Jefferies' interpretation is based on two misunderstandings. First, there is no theoretical reason why the spherical cavity expansion pressure should be higher than the cavity expansion pressure in a short cylinder at an equivalent strain. Jefferies states that the spherical case offers the 'least kinematic constraint', but fails to draw the obvious conclusion that it should therefore result in lower cavity expansion pressures than for more constrained problems. In fact actual cavity expansion pressures are the result of a subtle interplay between kinematic constraints, equilibrium of stresses and material properties which link stresses and strains. As is well known, spherical expansion pressures are higher than those for the expansion of a long cylinder (largely because of the differences in the equilibrium equations) in spite of the fact that spherical expansion is less constrained. The interplay between the equations is sufficiently complex for the case of expansion of a short cylinder that, without detailed analysis, no conclusions can be drawn as to whether it will require an expansion pressure greater or less than that for a sphere.

The second error is that Jefferies fails to account for the way in which the strength was derived from the finite element analyses (and is usually derived in engineering practice). It was derived from the slope of a plot of the expansion pressure against strain (on a logarithmic scale), as is explained on page 569 of the paper. Fig. 18 shows a comparison, for $I_{\mathrm{r}}=500$, of the exact cylindrical and spherical solutions with a finite element analysis of an infinitely long pressuremeter, which closely follows the exact solution for a cylinder, and with a finite element solution for $L / D=4$. At small strain the solution for the short pressuremeter is close to the cylindrical solution but at larger strain it comes closer

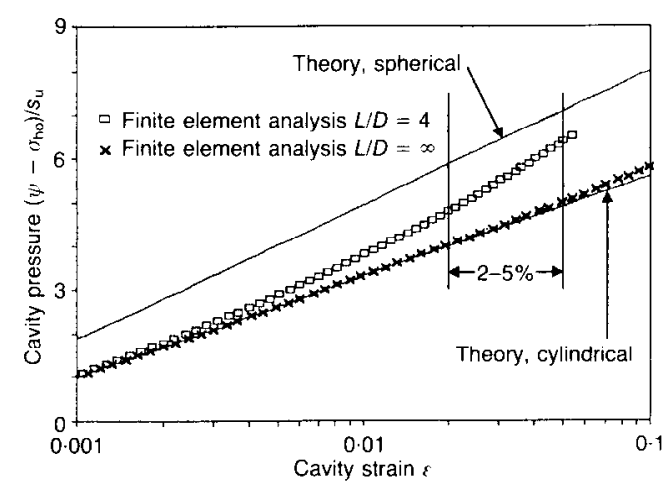

Fig. 18. Pressuremeter solutions for $I_{\mathrm{r}}=500$

to the spherical solution. Within the range of strain of $2-5 \%$, which was used to deduce $s_{u}$, the absolute value of the pressure is lower than that for a spherical cavity (as Jefferies would require, but is not in fact necessary), but the gradient of the curve is steeper-as it must be if the expansion approximates the cylindrical case at very small strain, but progressively approaches the spherical case as strain increases. Applying the conventional analysis therefore results in a significantly larger undrained strength than would be deduced from the same analysis of a spherical cavity; this is simply because the undrained strength is obtained from the gradient of the curve. Whether or not the absolute pressure is higher or lower than for the spherical case is irrelevant: it is the gradient of the curve that matters.

Jefferies' labelling of an 'Invalid solution space' is therefore erroneous and there is no 'error with the reported numerics' in the paper.

Jefferies continues to claim that the authors misunderstand the Tresca model. He states that the sloping portion ... can include workhardening plastic strain'. This is incorrect: the initial part of the stress-strain curve is purely elastic in the Tresca model. A soil will, of course, not exhibit this idealized behaviour-a point which is made clear in the paper.

Jefferies' statement that unique solutions (presumably for the material properties and in situ stresses) can be obtained only by using both expansion an contraction data for an SBP is incorrect: the contraction curve yields useful additional information, but strictly the cxpansion curve is sufficient. The approaches of Houlsby \& Withers (1988) and Jefferies (1988) result in similar mathematical expressions but interpret their use quite differently.

Jefferies claims that the authors suggest a range of $250<I_{\mathrm{r}}<800$, but these figures are not given anywhere in the paper. In fact the typical range 
suggested is $200<I_{\mathrm{r}}<500$. The authors agree, however, that when a compromise value of $G$ is used to represent the initial section of the stressstrain curve, lower values of $I_{\mathrm{r}}$ will also be encountered, often down to 100 and occasionally perhaps as low as 50 . Jefferies overstates his case by claiming that his figures support an $L / D$ effect of only one third of that suggested in the paper. His range of 55--250 for $I_{\mathrm{r}}$ would suggest that strengths would be overestimated by $9-29 \%$, rather than $25-43 \%$ for $I_{\mathrm{r}}=200-500$ as suggested in the paper.

The authors were careful to ensure that their results did not depend on use of triaxial undrained strength as a basis for comparison, and chose a theoretical model which results in a unique definition of undrained strength irrespective of shearing mode. They are not therefore guilty of the innocent simplicity implied by Jefferies. Nevertheless, most design practice in the UK has been based on the use of good quality triaxial compression testing as providing a reference value for the undrained strength. If strengths derived from other tests are to be used in connection with conventional design practice there is therefore a certain logic in using the triaxial compression test as a basis for comparison.

Jefferies supports his argument by reference to data from SBP tests at Bothkennar. These data are presented in Fig. 14 and suggest that when undrained strength is derived by conventional methods the trend line falls within the range of scatter of triaxial data. One cannot, however, draw conclusions about the general interpretation of in situ tests from the results obtained at a single site. The authors have examined data from the authoritative CIRIA report by Mair \& Wood (1987) and on London clay from Windle \& Wroth $(1977 \mathrm{a}, 1977 \mathrm{~b})$ in which self-boring pressuremeter data are compared with a trend line for plate loading tests and $98 \mathrm{~mm}$ dia. triaxial tests. This trend line is used as a reference value (a trend line for $38 \mathrm{~mm}$ triaxial tests is not used here because the slightly higher strengths it implies are probably due to the lack of fissures in the small samples). For 17 tests the peak and ultimate undrained strength is derived from the maximum and ultimate slopes of the $\psi: \ln (\Delta V / V)$ curve. The peak strengths vary from $21 \%$ to $113 \%$ higher than the triaxial and plate load test trend line, with an average of $67 \%$ higher. The ultimate values vary from $11 \%$ below to $61 \%$ above the line, with an average at $34 \%$ above.

Mair \& Wood (1987) also report data on three Italian clays from Ghionna, Jamiolkowski, Lacasse, Ladd, Lancellotta \& Lunne (1983). Again SBP tests are compared with triaxial compression tests (although triaxial extension, direct simple shear and field vane gave lower strengths).
For Porto Tolle clay the pressuremeter tests were from $9 \%$ below to $32 \%$ above triaxial compression, with a mean of $7 \%$ higher. For Panigaglia clay they were from $4 \%$ to about $170 \%$ higher (the latter figure being rather difficult to determine because of the scale of the figure) with an average of $61 \%$ higher. For Taranto clay the range was from $14 \%$ to at least $60 \%$ higher, with an average of $26 \%$ higher.

Even ignoring the peak strength data in London clay, these data suggest that pressuremeter tests typically give considerably higher strengths than triaxial tests, albeit with a wide variation. Accepting Jefferies' judgement that at Bothkennar there was no significant difference between triaxial and SBP strength, the average for the 71 tests from five sites shows that the pressuremeter gave a strength $24 \%$ higher than triaxial tests-just below the 25-43\% range suggested in the paper. The authors accept that, especially in the light of lower $I_{\mathrm{r}}$ values appropriate as compromise values of shear modulus, the typical overestimation of strength by the pressuremeter may be at the bottom end of the range they suggested previously, but cannot accept Jefferies' assertion that the magnitude of the difference is much smaller. The field data show a wide range of differences, and more than one effect may be the cause of these differences.

Jefferies gave an example at a recent international conference on advances in site investigation practice in which (when using his own method for accounting for $L / D$ effects) the estimated strength from a particular pressuremeter test dropped from $156 \mathrm{kPa}$ to $127 \mathrm{kPa}$ when the effect of finite length was taken into account. This implies an overestimation by $23 \%$. It seems that there is a great deal of evidence to support a much larger overestimation than the $10 \%$ suggested in Jefferies' discussion.

Shuttle repeats some of the errors made by Jefferies. She correctly states that a correction factor, which is a function of soil rigidity index, is suggested in the paper, but then incorrectly defines the factor as $\beta$ in equation (5). This definition is inconsistent with the approach used in the paper, where no suggestion is made that such a constant factor exists. Shuttle describes the assumption of such a constant factor as 'the authors' hypothesis', but in fact such a hypothesis was never made. The authors agree with her observation that $\beta$ as defined in equation (5) would be a function of cavity strain. This means that any way of interpreting pressuremeters which depends simply on applying a single factor to an entire pressureexpansion curve would be incapable of accounting properly for the effects of finite length.

Shuttle's definition would be appropriate if $s_{\mathrm{u}}$ were to be derived from equation (4) by using 
values of limit pressure, in situ horizontal stress and rigidity index deduced from a pressuremeter test. These quantities are all difficult to determine accurately from a pressuremeter test, and so this would be a very inaccurate way of deriving undrained strength. The procedure adopted in the paper uses the slope of the plot of pressure against strain (on a logarithmic scale). This procedure has the advantage that (for an ideal test) it does not depend on any of the above quantities, but gives the undrained strength directly. Even for a test which is not ideal, the paper showed that a correction factor was required which was only a function of rigidity index. Moreover, the factor depends only moderately on the rigidity index within the range of typical values.

Dr Shuttle quotes from the paper 'the strength derived from the pressuremeter would be about $25-43 \%$ higher than the actual strength of the soil', but omits the rider that this is for ' $I_{\mathrm{r}}$ values in the range 200-500'. Care is needed in the choice of $I_{\mathrm{r}}$ value: should the shear modulus used in its derivation be representative of the smallstrain value, or should it be a compromise value representing a wider range of strains? The latter is justified on the grounds that the parameters used in the simple elastic plastic model used for the soil should be chosen so that the model fits the real, highly non-linear, behaviour of the real soil over a reasonable range of strains. The result is a choice of modulus that underestimates stiffness at very low strain, and overestimates it at higher strain. Since the paper was written this author has come to the view that an appropriate compromise modulus could give an $I_{\mathrm{r}}$ value lower than 200, and agrees with Shuttle's implicit assumption that values as low as 50 may be relevant. This results in lower degrees of overestimation of the undrained shear strength, e.g. about $9 \%$ for $I_{\mathrm{r}}=50$ and the strength interpreted from the $2-5 \%$ section of the expansion curve (as in the original paper).

Shuttle comments about the corrections needed to the numerical calculations, and provides useful computations using a different code which achieved a close fit with theoretical results. Comparing the $I_{\mathrm{r}}$ values for $L / D=6$ in Table 4 with the figures for a strain range of $2-5 \%$ in Table 8 shows that except for $I_{\mathrm{r}}=1000$ the agreement is better than $0.4 \%$-remarkably good for two different numerical analyses. Only for the case of $I_{\mathrm{r}}=1000$ is the result obtained by Shuttle $6.5 \%$ different from that obtained in the paper. In the paper a $13.7 \%$ correction in this case had to be applicd and so it is likely that Shuttle's figure is the more reliable.
Shuttle also addresses the important issue that overestimation of strength is also a function of the choice of the range over which the pressureexpansion curve is fitted. Her analyses show that this overestimation could be minimized by choosing the strain range to be as early as possible in the test (subject to the constraint that it must be after yield).

In conclusion, the positions of the authors and the discussers are probably not as far apart as it seems at first. Experimental evidence suggests that, compared with the triaxial test, the SBP, when interpreted as infinitely long, overestimates undrained strength by anything from a negligible amount to about $60 \%$ - on occasions more. Most of the data reviewed suggest an overestimation of, say, $15-35 \%$, but the scatter is wide. The authors initially suggested a range of overestimation of $25-43 \%$, but in the light of lower rigidity indices being appropriate for some cases would move the lower bound of the estimate to, say, $9 \%$ (for $I_{\mathrm{r}}=$ 50). If a typical value is to be adopted then there is experimental evidence for an average of $24 \%$, slightly below range in the paper, and above the range of $10-18 \%$ suggested by Jefferies. However, this figure should properly be treated as a function of rigidity index and interpretation method.

\section{REFERENCES}

Ghionna, V., Jamiolkowski, M., Lacasse, S., Ladd, C. C., Lancellotta, R. \& Lunne, T. (1983). Evaluation of self-boring pressuremeter. Proceedings of international symposium on in-situ testing, vol 2, 294-301. Singapore: Nanyang Technological Institute.

Hight, D. W., Bond, A. J. \& Legge, J. D. (1992). Characterization of the Bothkennar clay: an overview. Géotechnique 42, No. 2, 303-347.

Houlsby, G. T. \& Withers, N. J. (1988). Analysis of the cone pressuremeter test in clay. Géotechnique 38, No. 4, 575-587.

Jefferies, M. G. (1988). Determination of horizontal geostatic stress in clay with self-bored pressuremeter. Can. Geot. J. 25, 559-573.

Mair, R. J. \& Wood, D. M. (1987). Pressuremeter testing: methods and interpretation. London: Butterworth.

Smith, I. M. \& Griffiths, D. V. (1988). Programming the finite element method, 2nd edn. New York: Wiley.

Windle, D. \& Wroth, C. P. (1977a). In situ measurement of the properties of stiff clays. Proc. $9 t h$ Int. Conf. Soil Mech., Tokyo 1, 347-352.

Windle, D. \& Wroth, C. P. (1977b). The use of a selfboring pressuremeter to determine the undrained properties of clays. Ground Engng 10, No. 6, Sept., $37-46$. 